\title{
Original article \\ Efficacy of latanoprost in management of chronic angle closure glaucoma
}

\author{
Kumar $\mathrm{S}^{1}$, Malik $\mathrm{A}^{2}$ \\ Singh $\mathrm{M}^{3}$, Sood $\mathrm{S}^{4}$ \\ ${ }^{1}$ Associate Professor, ${ }^{2}$ Assistant Professor, ${ }^{4}$ Professor, Department of Ophthalmology, Government Medical \\ College and Hospital, Chandigarh, ${ }^{3}$ Senior Lecturer, Department of Ophthalmology, Ram Manohar Lohia \\ Hospital, Delhi
}

\begin{abstract}
Background: Chronic angle closure glaucoma is often managed surgically.

Aim: To study the effect of Latanoprost $0.005 \%$ on intraocular pressure in subjects diagnosed as having chronic angle closure glaucoma.

Materials and methods: Forty patients participated in the study. Baseline examination included visual acuity, refraction, slit-lamp examination, intraocular pressure, anterior and posterior segment examination, gonioscopy and perimetry. Patients were treated with Latanoprost $0.005 \%$ once daily at bedtime. IOP was recorded at baseline, 2 weeks, 4 weeks, 8 weeks and 12 weeks after starting the treatment.
\end{abstract}

Results: The mean age of the study sample was 56.45 years (40-70 years). There were 18 males and 22 females in the study. Mean IOP at baseline was $24.55 \pm 3.63$. Mean IOP decreased to $17.27 \pm 3.19$ at 2 weeks, $15.27 \pm 3.07$ at 4 weeks, $14.60 \pm 3.06$ at 8 weeks and $14.47 \pm 2.66$ at 12 weeks. There was a statistically significant reduction in mean IOP $(41.03 \%)$ at 12 weeks as compared to those of the baseline IOP $(p=0.000)$. There was no significant difference in IOP reduction in eyes with different degrees of angle closure by peripheral anterior synechiae.

Conclusion: Latanoprost, a prostaglandin analogue, is effective in reducing IOP in chronic angle closure glaucoma patients and its efficacy is not affected by the degree of angle closure by peripheral anterior synechiae.

Key words: latanoprost, chronic angle closure glaucoma, intraocular pressure, peripheral anterior synechiae

\section{Introduction}

Glaucoma accounts for approximately 5.1 million of the blind people in the world, with more than half of these residing in Asia (Quigley, 1996; Thylefors et al 1995; Dandona et al 2000). Primary angle closure glaucoma

Received: 12.08.2008. Accepted: 14.11.2008

Correspondence and reprint requests to: Dr Archana Malik

Assistant Professor, Department of Ophthalmology

Government Medical College and Hospital, Chandigarh, India-160032

Email: drarchanag2002@yahoo.com

Fax: 0091-172-2607707
(PACG) is considered the most common form of glaucoma in Asia. Most glaucoma research has been focused on populations with a preponderance of primary open angle glaucoma (POAG), whereas treatment modalities for chronic angle closure glaucoma (CACG) remain less studied. In CACG, gradual asymptomatic angle closure results in diminished aqueous outflow through the angles and a subsequent rise of the intraocular pressure (IOP). Laser iridotomy remains the appropriate initial therapy for CACG to 
eliminate any element of pupillary block. Topical medications should only be added if iridectomy alone is insufficient to control IOP.

Latanoprost, a prostaglandin F2-alpha analogue, has proven to be an effective ocular hypotensive drug in subjects with POAG and ocular hypertension (Mishima et al 1996, Watson \& Stjernschantz 1996). Its main mechanism for reducing IOP is an increase in the uveoscleral outflow (Toris et al 1993). Very few studies have investigated the efficacy of latanoprost in reducing IOP in subjects with CACG (Aung et al 2005, Kook et al 2005, Chew et al 2002, Aung et al 2000, Hung et al 2000 , Chen et al 2006). The mechanism of action of latanoprost in eyes with closed angles is not established. It is not known whether the IOP-reducing effect of latanoprost varies with the extent of angle closure and whether latanoprost is effective in eyes with extensive peripheral anterior synechiae (PAS).

This study was carried out to see whether latanoprost was effective in reducing IOP in patients with CACG and whether the extent of PAS affected its action.

\section{Materials and methods}

Forty subjects participated in a 12-week study conducted across 2 centers in India. Informed consent was obtained from all subjects and the study was carried out in accordance with the World Medical Association's Declaration of Helsinki. Chronic angle-closure glaucoma was defined as optic neuropathy with a corresponding visual field defect, chronically raised IOP, an anterior chamber angle in which the trabecular meshwork was not visible for at least $180^{\circ}$ on gonioscopy, and with evidence of PAS in the angle. Patients included were those who were 40 years of age or older with unilateral or bilateral primary CACG, who had undergone peripheral iridotomy (PI) at least one month before, had IOP greater than $21 \mathrm{mmHg}$ after the PI, and current control with one or two pressure-reducing medications on two consecutive visits. Patients excluded were those who had undergone previous intraocular surgery (PI excluded)/trauma to the eye, patients on oral drugs known to affect the IOP, uveitis, causes of secondary angle closure and pregnant or nursing women.

At the pre-study visit, which took place 5 weeks be- fore the baseline visit, medical and ocular history was taken and subjects were assessed for eligibility. Visual acuity, refraction, IOP, anterior and posterior segment (cup-disc ratio) examination, gonioscopy (Shaffer's grading, degrees of PAS recorded) and perimetry were carried out. All existing IOP-lowering therapy was discontinued. Patients were required to complete a minimum washout period before the start of the study: 4 weeks for prostaglandins, 3 weeks for b-adrenergic antagonists, 2 weeks for adrenergic agonists, 5 days for cholinergic agonists and carbonic anhydrase inhibitors. The right eye was taken as the study eye in all subjects.

IOP was measured with a Goldmann applanation tonometer. At each time point, three measurements were performed in each eye, and the mean of three measurements was used in the statistical analyses. IOP was recorded at baseline, 2 weeks, 4 weeks, 8 weeks and 12 weeks after starting the treatment. Statistical analysis was done using one-way Anova test. Static and dynamic gonioscopy were performed at baseline and at 12 weeks. The examination was carried out at the lowest level of illumination that permitted a view of the angle and at high magnification. The drainage angle was graded according to Shaffer's grading in each quadrant and the degrees of PAS were recorded for each patient. Best-corrected Snellen visual acuity, systemic blood pressure, and pulse rate were determined at each visit, and a slit-lamp examination was performed. The presence of cells and flare in the anterior chamber was investigated during slit-lamp examination. Patients were instructed to administer one drop of Latanoprost at 8.00 PM everyday. Patients were instructed about punctal occlusion after administering the drops. Adverse events were monitored carefully throughout the study.

\section{Results}

In total, 40 patients entered the study. The mean age of the study sample was $56.45 \pm 7.87(40-70)$ years. There were $18(45.0 \%)$ males and $22(55.0 \%)$ females. Vertical cup-disc ratio, Shaffer's grade and degrees of peripheral anterior synechiae (PAS) are shown in Table 1. Mean IOP at baseline was $24.55 \pm 3.63(20-34)$ $\mathrm{mmHg}$. Mean IOP decreased to $17.27 \pm 3.19(12-26)$ at 2 weeks, $15.27 \pm 3.07(10-24)$ at 4 weeks, $14.60 \pm 3.06$ $(10-24)$ at 8 weeks and $14.47 \pm 2.66(10-22)$ at 12 weeks 
(Fig.1). There was 41.03\% $(10.07 \pm 0.71 \mathrm{mmHg})$ reduction of IOP at the end of 12 weeks. Repeated measure one-way Anova revealed statistically significant reduction in mean IOP at 12 weeks as compared to at baseline $\{$ Wilks' test $[\mathrm{F} \mathrm{4,156]}=48.773, \mathrm{p}=0.000\}$. The maximum reduction of IOP $(29.63 \%)$ was seen in the first 2 weeks, though significant decrease was seen thereafter also till the end point of study. Fig. 2 shows scatter plot of final IOP at 12 weeks versus baseline IOP.

\section{Tables}

Table 1. Vertical Cup-to-Disc Ratio, Shaffer's grade and degrees of PAS

\begin{tabular}{|c|l|}
\hline Parameter & $\begin{array}{l}\text { No. of Study } \\
\text { Subjects }(\mathbf{n}=\mathbf{4 0})\end{array}$ \\
\hline $\begin{array}{c}\text { Vertical Cup-to-Disc Ratio } \\
\text { Mean (SD) }\end{array}$ & $0.59(0.17)$ \\
Range & $0.3-0.9$ \\
\hline Shaffer's grade & \\
0 & $6(15.0)$ \\
0 -I & $7(17.5 \%)$ \\
I-II & $21(52.5 \%)$ \\
II-III & $6(15.0 \%)$ \\
III-IV & 0 \\
\hline Degrees of PAS & \\
$<180$ & $17(42.5 \%)$ \\
$180-270$ & $13(32.5 \%)$ \\
$>270$ & $10(25.0 \%)$ \\
\hline
\end{tabular}

Table 2. Decrease in IOP in eyes with different degrees of PAS

\begin{tabular}{|l|l|l|}
\hline $\begin{array}{l}\text { Degrees of } \\
\text { PAS }\end{array}$ & $\begin{array}{l}\text { IOP at } \\
\text { baseline }\end{array}$ & $\begin{array}{l}\text { IOP at 12 } \\
\text { weeks }\end{array}$ \\
\hline$<180$ & $25.88 \pm 4.35$ & $13.64 \pm 2.14$ \\
\hline $180-270$ & $24.38 \pm 2.02$ & $14.61 \pm 3.15$ \\
\hline$>270$ & $22.50 \pm 3.17$ & $15.70 \pm 2.49$ \\
\hline
\end{tabular}

On comparing the decrease in IOP in eyes with different degrees of PAS, no significant difference was found between the three groups by repeated measure one-way Anova $\{$ Wilks' test $[\mathrm{F} 8,68]=1.744, \mathrm{p}=0.104\}$. Table 2 and Fig. 3 depict the decrease in IOP in the three groups with different degrees of PAS.

\section{Figures}

Fig.1 Line graph showing IOP change from baseline to 12 weeks

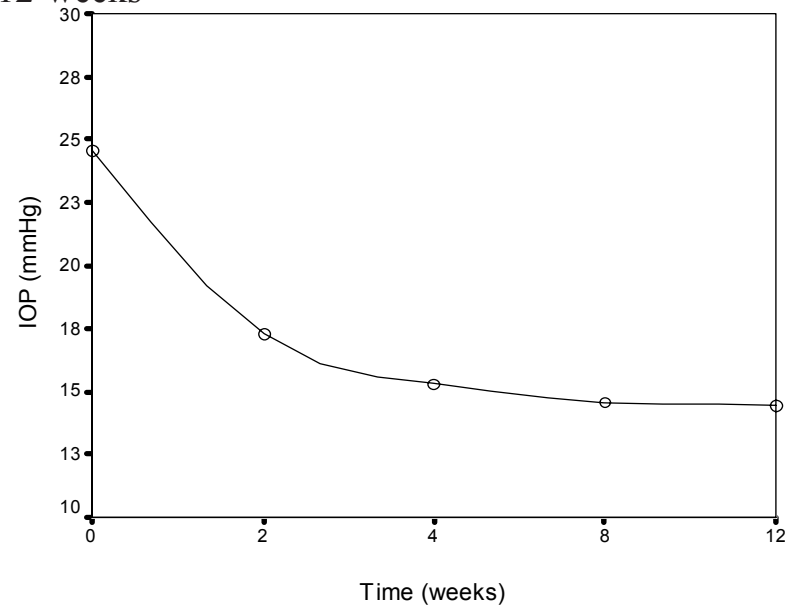

Fig.2 Scatter plot showing final IOP $(\mathrm{mmHg})$ at 12 weeks versus baseline IOP ( $\mathrm{mmHg}$ )

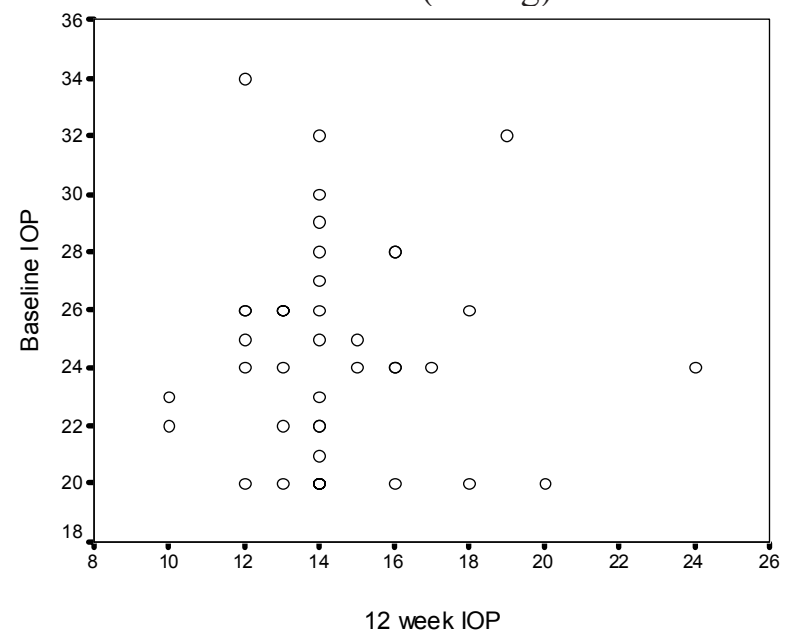

Fig.3 Bar diagram depicting change of IOP (mmHg) with different degrees of peripheral anterior synechiae (PAS)

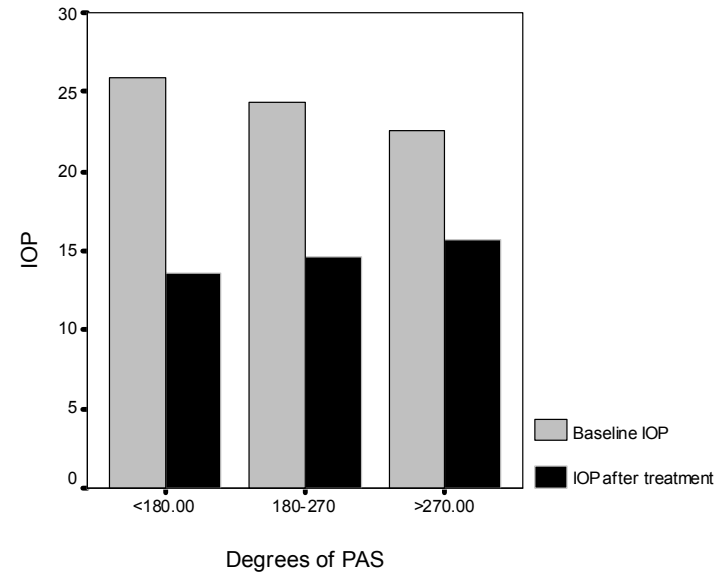


Latanoprost was well tolerated in our patients. Most of the adverse events were reported as mild. The most common ocular adverse events found were that of conjunctival hyperemia and ocular discomfort.

\section{Discussion}

This study shows that Latanoprost is effective in reducing IOP in CACG patients and its efficacy is not affected by the degree of angle closure by PAS. Though higher IOP reduction was seen in eyes with lesser degrees of PAS, it was not statistically different from those with greater degrees of PAS.

Laser iridotomy is the still the first line of management in CACG to eliminate any element of pupillary block and topical medications should only be added if the iridectomy alone is insufficient to control IOP.

The exact mechanism of action of latanoprost in eyes with closed angles is not known. The main mechanism of action of prostaglandin analogs in the reduction of IOP has been shown to be increased uveoscleral outflow of aqueous humor (Toris et al 1993). They act on specific ciliary muscle prostanoid receptors and thus lead to increased biosynthesis of matrix metalloproteinases, which can cleave extracellular matrix components (Lindsey et al 1997). This alters the collagen content in the ciliary muscle, thereby reducing the hydraulic resistance in the uveoscleral pathway. In CACG, latanoprost may gain access to the ciliary body through the still-open part of the anterior chamber angle to increase uveoscleral outflow. It is also possible that it is able to increase outflow of aqueous through the PAS in closed angles, explaining its efficacy in eyes with up to 12 clock hours of PAS. Another possibility is that latanoprost gains access to the uveoscleral outflow through other routes such as through the posterior chamber between the iris and lens, the iris root itself, or the sclera. Evidence for this is suggested by the finding that topical prostaglandin treatment induces matrix metalloproteinases and reduces collagens in the sclera and iris root (Sagara et al 1999).

The preliminary study by Chew et al (2002) in CACG patients demonstrated a $34.2 \%$ decrease in IOP at 2 weeks with latanoprost as opposed to $29.63 \%$ reduction seen in our study.
Our study was comparable to a study done by Aung T et al (2005). Demographic characteristics were similar in both the studies. Mean vertical CD ratio was $0.59 \pm$ 0.17 as compared to $0.61 \pm 0.2$ in our study. $80 \%$ of our patients had Shaffer's grade less than or equal to 2 while in theirs $72 \%$ patients had $<$ grade 1 ; and 10 patients in our study had more than 270 degrees of PAS as compared to 15 patients in their study having more than 8 clock hours of PAS. The mean IOP levels at baseline and at week 12 were $24.55 \pm 3.63$ and $14.47 \pm 2.66$ respectively in our study (corresponding values in their study were $25.2 \pm 6.0 \mathrm{mmHg}$ and $17.7 \pm 5.3 \mathrm{~mm} \mathrm{Hg}$ ). Mean reduction of IOP was considerably higher in our study (41.03\%) as compared to their study (29.7\%). No particular reason for this could be elucidated. Mean IOP reduction in patients with $>270$ degrees of PAS was $30.2 \%$ in our study which was comparable to $31 \%$ in patients with $>8$ clock hours of PAS in their study. They noticed a higher hypotensive response in eyes with greater angle closure. Though there was significant decrease in IOP in eyes with variable degrees of PAS, our study showed a higher IOP reduction in patients with lesser degrees of PAS, but the difference was not statistically significant.

\section{Conclusion}

Our study demonstrates that latanoprost does reduce IOP in CACG patients and significant reduction is also seen in patients with $>270$ degrees of PAS showing that the amount of angle closure does not reduce the action of latanoprost.

\section{Acknowledgements}

No grants or sponsorships have been requisitioned for this study. The authors do not have any proprietary or financial interest in any procedure or product mentioned in this manuscript.

\section{References}

Aung T, Chan YH, Chew PTK, EXACT Study Group (2005). Degree of angle closure and the intraocular pressure-lowering effect of latanoprost in subjects with chronic angle-closure glaucoma. Ophthalmology 112:267-271.

Aung T, Wong HT, Yip CC et al (2000). Comparison of the intraocular pressure-lowering effect of latanoprost and timolol in patients with chronic angle closure glaucoma: a preliminary study. Ophthalmology 
107:1178-83.

Chen MJ, Chen YC, Chou CK, Hsu WM(2006). Comparison of the effects of latanoprost and travoprost on intraocular pressure in chronic angle-closure glaucoma. J Ocul Pharmacol Ther 22:449-54.

Chew PTK, Hung PT, Aung T(2002). Efficacy of Latanoprost in reducing intraocular pressure in patients with primary angle-closure glaucoma. Survey of Ophthalmology 47:125-128.

Dandona L, Dandona R, Mandal P et al(2000). Angle-closure glaucoma in an urban population in southern India. The Andhra Pradesh Eye Disease Study. Ophthalmology 107:1710-6.

Hung PT, Hsieh JW, Chen YF, Wei T(2000). Efficacy of latanoprost as an adjunct to medical therapy for residual angle-closure glaucoma after iridectomy. J Ocul Pharmacol Ther 16:43-7.

Kook MS, Cho HS, Yang SJ, Kim S, Chung J (2005) . Efficacy of Latanoprost in Patients with Chronic Angle-Closure Glaucoma and No Visible Ciliary-Body Face: A Preliminary Study. Journal of Ocular Pharmacology and Therapeutics 21: 75-84.

Lindsey JD, Kashiwagi K, Kashiwagi F(1997). Prostaglandin action on ciliary smooth muscle extracellular matrix metabolism: implications for uveoscleral outflow. Surv Ophthalmol 41:53.

Mishima HK, Masuda K, Kitazawa Y, et al.(1996) A comparison of latanoprost and timolol in primary open-angle glaucoma and ocular hypertension. A 12-week study. Arch Ophthalmol 114:929 -32.

Quigley HA(1996). Number of people with glaucoma worldwide. Br J Ophthalmol 80:389-93.

Sagara T, Gaton DD, Lindsey JD et al (1999). Topical prostaglandin $\mathrm{F} 2$ alpha treatment reduces collagen types I, III, and IV in the monkey uveoscleral outflow pathway. Arch Ophthalmol 117:794-801.

Thylefors B, Negrel AD, Pararajasegaram R, Dadzie KY(1995). Global data on blindness. Bull World Health Organ 73:115-21.

Toris CB, Camras CB, Yablonski ME (1993). Effects of $\mathrm{PhXA41}$, a new prostaglandin $\mathrm{F} 2$ alpha analogue on aqueous humour dynamics in human eyes. Ophthalmology 100:1297-304.

Watson P, Stjernschantz J, Latanoprost Study Group (1996). A six month randomized, double-masked study comparing latanoprost with timolol in openangle glaucoma and ocular hypertension. Ophthalmology 103:126-37. 